
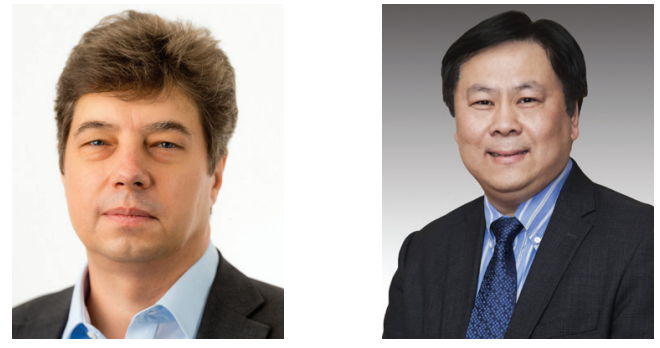

\title{
Advanced Photonics: exciting news all around
}

Time flies. We remember vividly how we worked on the first issue of Advanced Photonics, chasing the authors and the reviewers, choosing a journal cover, checking proofs. It was more than three years ago. Three volumes and many articles were published since then. The journal is coming of age. In the past year, Advanced Photonics was accepted to Clarivate's Science Citation Index Expanded (Web of Science) and to Elsevier's Scopus. This means it should receive the first impact factor in June 2022. While many views have been expressed about the Journal Impact Factor ${ }^{\mathrm{TM}}$, it is nevertheless the indicator of how articles are read and used by researchers in the field, showing that the journal is a trusted source of information. With the inclusion in relevant journal databases and indices, the published articles are now easy to find and enjoy. We are glad we can ensure this service for our readers and authors.

It was a busy year. In response to the significantly increased number of submissions, we enhanced our Editorial Board with two new members, Prof. Changhuei Yang and Prof. Yu-Jung Lu. We published a special collection on Quantum Technologies under the editorship of Mario Agio and Chaoyang Lu, which covered the recent advances involving photonics in this rapidly expanding area. Traditional annual Editors-in-Chief best paper award was expanded in 2021 to cover not only original articles abut also reviews. Very many congratulations to the winning articles: "Terahertz surface plasmonic waves" in the review category and "Enhanced light-matter interactions in dielectric nanostructures via machine-learning approach" in the original article category.

We are excited to announce the launch of our sibling journal Advanced Photonics Nexus to help our authors to find an appropriate home for their high-quality work fast. The Nexus will begin accepting submissions shortly with a first issue planned for the second half of the year. This of course will be a Gold Open Access journal with article processing charges waived until later.

We are looking forward to meeting our readers and authors in 2022 with many exciting conferences planned to present Advanced Photonics, including stands at Photonics West and Photonics Europe exhibitions, at the Gordon Plasmonics \& Nanophotonics conference, and many others. All your thoughts and suggestions on the future of Advanced Photonics are very much appreciated and we are looking forward to talking to you.
Anatoly Zayats

Co-Editor-in-Chief

Xiao-Cong (Larry) Yuan Co-Editor-in-Chief

(C) The Authors. Published by SPIE and CLP under a Creative Commons Attribution 4.0 International License. Distribution or reproduction of this work in whole or in part requires full attribution of the original publication, including its DOI.. [DOI: 10.1117/1 AP.4.1.010101] 